On Education and Distribution

Author(s): P. G. Hare and D. T. Ulph

Source: The Journal of Political Economy, Vol. 87, No. 5, Part 2: Education and Income

Distribution, (Oct., 1979), pp. S193-S212

Published by: The University of Chicago Press

Stable URL: http://www.jstor.org/stable/1829914

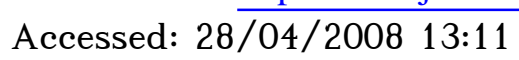

Your use of the JSTOR archive indicates your acceptance of JSTOR's Terms and Conditions of Use, available at http://www.jstor.org/page/info/about/policies/terms.jsp. JSTOR's Terms and Conditions of Use provides, in part, that unless you have obtained prior permission, you may not download an entire issue of a journal or multiple copies of articles, and you may use content in the JSTOR archive only for your personal, non-commercial use.

Please contact the publisher regarding any further use of this work. Publisher contact information may be obtained at http://www.jstor.org/action/showPublisher?publisherCode=ucpress.

Each copy of any part of a JSTOR transmission must contain the same copyright notice that appears on the screen or printed page of such transmission.

JSTOR is a not-for-profit organization founded in 1995 to build trusted digital archives for scholarship. We enable the scholarly community to preserve their work and the materials they rely upon, and to build a common research platform that promotes the discovery and use of these resources. For more information about JSTOR, please contact support@ jstor.org. 


\title{
On Education and Distribution
}

\section{P. G. Hare}

University of Stirling

\author{
D. T. Ulph
}

University College London

This paper examines the rules governing the optimal distribution of educational resources originally developed by Arrow, in the context of a model in which some attempt is made to provide a rationale for government expenditure. Thus while there is a private market providing a perfect substitute for government education, if the government cannot adopt the lump-sum taxation which would make exclusive reliance on the private market optimal, but has to rely on an income tax for redistribution, then an optimally chosen scheme of educational provision by the government will powerfully reinforce the redistributive effect of the income tax.

\section{Introduction}

Two of the perennial debates in the field of education concern the role that education should play in bringing about a more egalitarian society, and the extent to which education should be provided through the public sector rather than through the private market. While these are separate issues, they are clearly related in that it is often claimed that education can only play a positive role in bringing

This paper was prepared for the U.S./U.K. conference on human capital and income distribution held at King's College, Cambridge, March 1978. It has been substantially revised in the light of comments on the earlier version presented at the conference and further comments from referees of this Journal. We should like to thank all those who provided us with feedback on the paper, but freely accept responsibility for any errors that may remain.

[Journal of Political Economy, 1979, vol. 87, no. 5, pt. 2]

(C) 1979 by The University of Chicago. $0022-3808 / 79 / 8752-0009 \$ 01.58$ 
about a more just society if it is provided through the government (see, e.g., Tawney 1938, pp. 144-47).

In this paper we present a simple model in which these issues can be explored simultaneously. More precisely, we present a model in which there is the possibility of both public and private provision of education and in which, moreover, the private market is assumed to be "perfect" in that the only reason for wanting to use the public sector is to achieve distributional objectives. On the other hand, education is not the only distributional tool at the government's disposal, for we assume there is also an income tax which can alter the distribution of income both directly (by transferring income from some to others), but also indirectly, by influencing the amount of private education people choose to buy and hence the amount of income they earn. The government's problem is to determine the structure of the income tax and both the amount and the distribution of public education. By exploring the nature of the solution to this problem we hope to throw some light on the two questions we began with.

Our model has its roots in two different strands of the recent public finance literature. The first strand originates in the paper by Arrow (1971) in which he sought to characterize the optimal distribution of a given amount of public expenditure among a population of individuals who differed in their ability to benefit from this expenditure. One feature of Arrow's work is that he conducts his analysis "in some isolation from the general problem of income distribution" and consequently considers neither the question of whether the government should be using taxation rather than expenditure as a redistributive instrument, ${ }^{1}$ nor the related issue of why the expenditure is being provided through the public sector in the first place.

It would clearly be desirable to embed the Arrow approach in a more general framework in which the reasons for having at least some education publicly provided and for allocating this education differentially as part of an overall redistributive policy were fully modeled.

There are clearly a number of reasons that can be given for public provision: that education has some public good aspects, that capital markets are imperfect, etc. While we do not wish to discount such arguments, the approach which lies behind this paper rests on distributional considerations and is as follows: If we ignore capital market imperfections and any public goods aspects of education, then we know that if lump-sum taxation is available the government can achieve the first-best optimum. It does this by setting the lump-sum taxes at the right levels, leaving private markets to handle the alloca-

\footnotetext{
${ }^{1}$ Some aspects of this question are explored in the papers by Bruno (1976) and Ulph (1977).
} 
tion of private goods, in particular, education. In such an economy all redistribution is handled through the tax system.

However, lump-sum taxation is not available, and the government has to resort to distortionary income and commodity taxation. Here we make contact with the second strand in the recent public finance literature-the work on optimal taxation. While government expenditure has been considered in some of the models in this area, it has typically taken the form of an exogenously given revenue requirement (e.g., in the work of Feldstein [1973] and Stern [1976]) or as financing the provision of pure public goods (Diamond and Mirrlees 1971). What is not considered is the possibility of direct provision of private goods in the sense that the government simply gives people a certain amount of a given commodity free of charge, or, alternatively, compelling people to buy a certain minimum amount, at the same time providing them with a grant to make the purchase. It is an easy exercise to show that in some fairly simple models of optimal income taxation with private educational provision (such as the one we develop in Section III of this paper), the introduction of such a minimum compulsory purchase of education is a welfare improvement. ${ }^{2}$ Unfortunately, for reasons which will emerge when we have considered this model in detail, and to which we will return in the concluding section of the paper, we have as yet found it quite hard to say much more than this, and in particular to say how this minimum provision (and the tax schedule) should vary with any observable characteristics of individuals (the original Arrow question). ${ }^{3}$

Accordingly, in this paper we present a much simpler model with which we can study the interaction of tax and expenditure policies when there is both public and private provision of education, though the model itself does not contain the full rationale for such government provision. Thus we assume that there is a single attribute"ability to benefit from education"-which can be observed by the government for the purposes of allocating education but not for levying taxes. While clearly unsatisfactory as part of a model which seeks to give a full justification of government provision of education, the assumption can nevertheless be justified as a fairly realistic description of some aspects of education and tax policy (e.g., special

${ }^{2}$ All that is required is to show that the constraint will bind for those with a positive marginal tax rate. For then the introduction of the constraint will raise net revenue for the government, which has positive social value, while having no effect on welfare to the first order of approximation. Kevin Roberts (1978) has shown that this argument also goes through in the case where people face a zero marginal tax rate, though in this case it is necessary to consider second-order approximations to the gains.

${ }^{3}$ Of course, if the tax system can be related to observed variables there would have to be some unobserved variables so that the problem does not reduce once again to the first-best optimum. 
provision of educational resources to inner-city areas, without a corresponding tailoring of the income-tax system to residential location).

Two additional simplifying assumptions should be pointed out at the outset. First, as indicated, we are ignoring capital market imperfections, and so the model is collapsed to a timeless or one-period model. Without modeling such imperfections fully, it would be possible to reintroduce these considerations into the model by having some other unobservable "family background" variable which influences the costs of achieving any given wage rate. As indicated above, this is precisely the type of model we had started out with, and as yet we can say little about the nature of its solution.

Second, we assume that individuals supply a fixed number of hours of work. This has two implications. The first is that by a combination of the two policies the government can still achieve the first-best optimum. However, as this involves 100 percent marginal tax rates, an option that would certainly not be optimal without this assumption, we have to remove this first-best solution by imposing an upper bound on the marginal tax rate. The second implication is that we cannot allow the possibility of having educational expenditure offset against income for tax purposes. If we allow this possibility, then as long as marginal tax rates are less than 100 percent the individual will choose the amount of education which maximizes his gross earnings net of education costs. This amount will be independent of the particular tax schedule chosen. But then with effectively fixed labor supply, it will always be an improvement to bring marginal tax rates as close to 100 percent as possible. Consequently, we get no interesting solutions to the problem. Both of these complications can be removed by dropping the fixed-labor-supply assumption, and in a forthcoming paper (Hare and Ulph 1979) we have been able to extend our analysis to this case. Many of the conclusions of this model, however, continue to go through.

Having set out some of the broad features of the model, let us look at it in more detail.

\section{The Model}

We consider a population of individuals who differ in their "ability to benefit from education." This ability is assumed to be measured by the scalar $n$.

Ability is distributed in the population with density function $f(n)$. We assume $f(n)>0$ for $\boldsymbol{n} \leqq n \leqq \bar{n}$, where $\boldsymbol{n}>0, \bar{n} \leqq \infty$. The restriction $\bar{n}>0$ is not crucial to the general analysis we undertake, but when we come to take a specific form for our education equation, some of the properties we want to impose on the function will not be valid if $n=0$. 
If an individual of ability $n$ receives an amount of education $e$, his wage rate, $w$, is given by the equation

$$
w=\phi(n, e) .
$$

We assume that for all $n, \boldsymbol{n} \leqq n \leqq \bar{n}$, and for all $e \geqq 0$,

$$
\phi_{n}>0, \phi_{e}>0, \phi_{e e}<0, \phi_{n e}>0 .
$$

The first two assumptions are that both ability and education are productive, the third that there are diminishing marginal returns to education, while the final assumption states that those of higher ability benefit more at the margin as well as in absolute terms from any given amount of education. This is in many ways the crucial sense in which people benefit more from education.

We assume that everyone works the same fixed number of hours, and consequently choose units so that $w$ measures an individual's gross earnings.

For most of the analysis it will be convenient to invert $\phi(\cdot)$ and work with the cost function

$$
e=\psi(n, w),
$$

which gives the cost, in terms of educational resources, of giving an individual of ability $n$ a wage rate $w$. The function $\psi(\cdot)$ is defined implicitly through the identity

$$
w \equiv \phi[n, \psi(n, w)] .
$$

From (4) it follows that $\psi_{w}=1 / \phi_{e}>0, \psi_{n}=-\phi_{n} / \phi_{e}<0, \psi_{w w}=-\phi_{e e} / \phi_{e}^{3}$ $>0, \psi_{n w}=-\left(\phi_{n e} \psi_{w}+\phi_{e e} \psi_{w} \psi_{n}\right) / \phi_{e}<0$. Thus total and marginal costs are rising in the wage rate, falling in ability.

The government chooses an income-tax schedule which we characterize by the function

$$
x=z(w),
$$

giving the amount of net income, $x$, received by an individual whose gross income is $w$.

The government also chooses how much education it will give to an individual of ability $n$. This education is a perfect substitute for private education, and the individual can choose, if he wishes, to augment the government provision by purchasing additional education privately. However, it is crucial to the model that the government provision cannot be sold, since otherwise government education is effectively a lump-sum subsidy. We discuss this point more fully in the concluding section.

In deciding whether or not to purchase private education, the 
individual is assumed to make the decision which maximizes consumption. That is, he chooses $w$ so as to

$$
\max _{w \geqq 0}[z(w)+g(n)-\psi(n, w)]
$$

subject to $\psi(n, w) \geqq g(n)$.

Notice that this formulation of the budget constraint embodies the further assumption that educational resources are being measured in the same units as consumption or income.

Finally, the government has to choose $z(w)$ and $g(n)$ so as to maximize the social welfare function

$$
W=\int_{n}^{\bar{n}} u[c(n)] f(n) d n
$$

subject to the resource constraint that total outlays on consumption and education do not exceed total income,

$$
\int_{n}^{\bar{n}}\{c(n)+\psi[n, w(n)]\} f(n) d n \leqq \int_{n}^{\bar{n}} w(n) f(n) d n,
$$

and subject also to the requirement that the functions $c(n), w(n)$ arise from the individual maximization decisions in (6).

The solution to this problem will be investigated in detail in Section V. Before considering that, it will be useful to look at the optimal structure of each policy in isolation.

\section{Income Taxation with No Public Provision of Education}

In this case, the individual's problem is to choose his wage and consumption so as to

$$
\max _{c, w \geq 0} c \quad \text { such that } c+\psi(n, w) \leqq z(w) .
$$

The first-order characterization of the solution is

$$
z^{\prime}(w)-\psi_{w} \leqq 0, \quad w \geqq 0 .
$$

At an interior solution, $z^{\prime}(w)>0$, so the marginal tax rate is less than 100 percent, while, differentiating the budget constraint, we find

$$
c^{\prime}+\psi_{n}+\psi_{w} w^{\prime}=z^{\prime} w^{\prime},
$$

that is,

$$
c^{\prime}=-\psi_{n} .
$$

This is just an envelope result stating that as long as each individual is choosing his optimal income, consumption increases with ability to the extent that the more able need spend less on education to achieve the same wage rate as the less able. 
The government's problem is to choose $c(\cdot), w(\cdot)$ so as to

$$
\begin{aligned}
& \max \int_{n}^{\bar{n}} u[c(n)] f(n) d n \\
& \quad \text { such that } \int_{n}^{\bar{n}}\{c(n)+\psi[n, w(n)]-w(n)\} f(n) d n \leqq 0,
\end{aligned}
$$

and subject also to the constraint that $c(\cdot)$ and $w(\cdot)$ arise as solutions to the individual maximization problem.

Before considering the solution to the government problem, notice that we can rewrite the individual and government problems in the following way:

Let $v(x, w, n) \equiv u[x-\psi(n, w)]$ be the utility an $n$ man achieves from net income $x$ when he has to spend the amount $\psi(n, w)$ to earn gross income $w$. Then (8) becomes

$$
\max _{x, w} v(x, w, n) \quad \text { such that } \quad x \leqq z(w),
$$

while (11) becomes

$$
\max \int_{n}^{\bar{n}} v[x(n), w(n), n] f(n) d n \quad \text { such that } \int[x(n)-w(n)] f(n) d n \leqq 0,
$$

and the constraint that $x(n), w(n)$ maximize $v(\cdot)$.

In this formulation the model is now identical to the original Mirrlees model, and we can carry over all the general conclusions about the nature of the optimal tax schedule for that model. Thus we know that the marginal tax rate will be zero at the top end of the distribution. To see what happens at the bottom end of the distribution we need to consider what happens to the marginal rate of substitution $\left(-v_{w} / v_{x}\right)$ when $w=0$.

From the definition of $v(\cdot)$, it is easily seen that, when $w=0,-v_{w} / v_{x}$ $=\psi_{w}(n, 0)$. If this is independent of $n$ (which is certainly the case for a function such as $\phi=n e^{\beta}$ ), then the phenomenon of bunching can arise, and it is no longer possible to conclude that the marginal tax rate must be zero at the bottom end of the income range.

In fact, the only way in which bunching can arise in this model is if the marginal tax rate is 100 percent at the bottom end of the income range, thus causing individuals with ability lying in some finite interval $(\boldsymbol{n}, \boldsymbol{n}+\delta)$ to choose zero education (and income). In the Appendix we show that with the assumptions we have made about $\phi$ and $z$, this possibility is ruled out. Consequently, individuals will always choose $w$ $>0$, and we can now characterize the government's problem as

$$
\begin{aligned}
& \max _{c(\cdot), w(\cdot)} \int_{n}^{\bar{n}} u[c(n)] f(n) d n \\
& \text { such that } \int_{n}^{\bar{n}}\{c(n)+\psi[n, w(n)]-w(n)\} f(n) d n \leqq 0, \\
& c^{\prime}(n)=-\psi_{n}[n, w(n)] .
\end{aligned}
$$


Introducing Lagrange multipliers $\lambda$ and $\alpha(n)$ for these constraints and integrating by parts, we obtain the following first-order conditions:

$$
\begin{gathered}
{\left[u^{\prime}(c)-\lambda\right] f(n)=\alpha^{\prime}(n),} \\
\lambda\left(1-\psi_{w}\right) f(n)=-\alpha(n) \psi_{n w},
\end{gathered}
$$

along with the transversality conditions $\alpha(\boldsymbol{n})=\alpha(\bar{n})=0$.

Now $\left(1-\psi_{w}\right)$ is just the marginal tax rate faced by an $n$ man, so (13) combined with the transversality conditions confirms that the marginal tax rates will be zero at each end of the income range. Moreover, we must have $u^{\prime}[c(\boldsymbol{n})]>\lambda$, since otherwise, as $c$ rises and $u^{\prime}(c)$ falls steadily with $n,(12)$ would imply that $\alpha$ falls continuously, so violating the end-point condition $\alpha(\bar{n})=0$. Thus $\alpha$ starts at zero, increases until $u^{\prime}(c)$ falls to $\lambda$, and then falls to zero again. This implies a positive marginal tax rate on the interior of $(\boldsymbol{n}, \bar{n})$. Other things being equal, this behavior of $\alpha$ would be reflected in the behavior of the marginal tax rate, but at this level of generality there is not much that can be said about the behavior of $f(\cdot)$ and $\psi_{m w}$.

Let us turn attention then to the nature of the optimal education choices that individuals make. By considering the first-order conditions for individual choice (9) we can see that if an $n$ man were to choose the same wage as an $n^{\prime}$ man, $n^{\prime}<n$, then given that $\psi_{m w}<0$ (when $w>0$ ), it follows that $z^{\prime}\left(w^{\prime}\right)-\psi_{w}\left(n, w^{\prime}\right)>0$; so provided $\left(z^{\prime}-\right.$ $\psi_{w}$ ) is falling in $w$ (as the second-order conditions for a maximum require), the $n$ man will choose a higher wage than the $n^{\prime}$ man. Thus the optimal policy is, in Arrow's terminology, output regressive.

To see what is happening to educational inputs, we have

$$
\frac{n e^{\prime}}{e}=\frac{n \psi_{n}}{\psi}+\frac{w \psi_{w}}{\psi} \cdot \frac{n w^{\prime}}{w},
$$

so that $e^{\prime}>0$ if and only if:

$$
m w^{\prime} / w>-\frac{n \psi_{n} / \psi}{w \psi_{w} / \psi} .
$$

For the particular case $\psi=n^{-1 / \beta} w^{1 / \beta}$, this becomes $\left(n w^{\prime} / w\right)>1$, so that a 1 percent increase in ability must bring about a more than 1 percent increase in the wage rate.

By differentiating (10) totally with respect to $n$, we find, after some manipulation, that

$$
\frac{n w^{\prime}}{w}=-\frac{n \psi_{m w} / \psi_{w}}{\left(w \psi_{w w}\right) / \psi_{w}-\left(w z^{\prime \prime}\right) / z^{\prime}},
$$

so, remembering that the denominator in (15) is positive if the second-order conditions for a maximum hold, (14) becomes 


$$
\frac{w z^{\prime \prime}}{z^{\prime}}>\frac{w \psi_{w w}}{\psi_{w}}-\frac{w \psi_{n w}}{\psi_{n}}
$$

For the special case $\psi=n^{-1 / \beta} w^{1 / \beta}$, (16) becomes $w z^{\prime \prime} / z^{\prime}>-1$.

In other words, as long as the marginal tax rate is not rising too fast, educational inputs will be increasing in ability. Given our previous discussion, this suggests that if there is going to be a range of ability over which educational inputs are falling, it is likely to occur at the bottom end of the ability scale.

Now by differentiating (13) and substituting (12), one can show that for the case where $\psi=n^{-1 / \beta} w^{1 / \beta}$,

$$
\begin{aligned}
(1-\beta) \frac{n w^{\prime}}{w}=\psi_{w}+\beta\left(1-\psi_{w}\right) & \frac{n f^{\prime}(n)}{f(n)} \\
& -\psi_{w}\left[\frac{u^{\prime}(c)}{\lambda}-1\right]+\left(1-\psi_{w}\right)(1+\beta),
\end{aligned}
$$

so that for $n \approx \boldsymbol{n}$ and consequently $\psi_{w} \approx 1$,

$$
\frac{n w^{\prime}}{w} \approx \frac{1-\left(u^{\prime}\{[c(\boldsymbol{n})] / \lambda\}-1\right)}{1-\beta}
$$

which can be either greater or less than one, and so unfortunately does not enable us to say much more about the nature of the optimal educational outlays without computing the full solution to the problem. Some preliminary calculations we have performed suggest that it is certainly possible to find examples where the optimal educational input will be falling over the initial ability range, so that educational inputs will be progressive.

We conclude, then, that when the government can control educational expenditure solely through an income tax, expenditure is always output regressive, and if input progressive at all will only be so at the lower end of the ability range.

\section{No Income Taxation: Public Provision of Education}

We assume now that the government has a fixed amount, $G$, of education which it can allocate among individuals of different abilities.

Suppose an individual of ability $n$ is allocated an amount of education, $g$; he then chooses the amount of private education he wishes to purchase, $e$, so as to

$$
\max _{e \geqq 0} u[\phi(n, g+e)-e] .
$$

Let $v(n, g)$ be the maximum utility he achieves. Thus

$$
v(n, g) \equiv u[\phi(n, g+\hat{e})-\hat{e}]
$$


where $\hat{e}$ is defined by

$$
\phi_{E}(n, g+\hat{e}) \leqq 1, \quad \hat{e}>0 .
$$

It is easily seen that

$$
v_{g}=u^{\prime}(c) \cdot \phi_{E} .
$$

The government's problem is to choose $g(n)$ so as to

$$
\max \int_{n}^{\bar{n}} v[n, g(n)] f(n) d n \text { such that } \int_{n}^{\bar{n}} g(n) f(n) d n \leqq G .
$$

This problem is formally identical to the original Arrow problem (Arrow 1971), although with the additional structure we have given the problem through the construction of $v(\cdot)$ in (18), the solution too has more structure. Since the detailed analysis of this problem has been set out elsewhere (Hare and Ulph 1978), we will simply report the main conclusions.

The solution to the problem (21) can be characterized by the conditions

$$
v_{g}[n, g(n)] \leqq \lambda, \quad g(n) \geqq 0,
$$

which in the light of (20) can be written

$$
u_{c}[c(n)] \cdot \phi_{E}[n, g(n)+e(n)] \leqq \lambda .
$$

It is then easy to see that the full solution has the property that the ability range is split into three separate intervals, within each of which the solution has different characteristics.

First interval: In this interval, $g(n)>0$ and $e(n)=0$, that is, these individuals consume only public education. The phase is characterized by $\psi_{E}<1, u^{\prime}(c)>\lambda$. This phase covers the lowest part of the ability range, as well as the lowest part of the consumption distribution. Since there is no use made of the private market for these cases, the characteristics of the solution are as given by Arrow (1971), Bruno (1976), and Ulph (1977). Thus those of higher ability will have greater earnings and hence consumption. On the other hand, educational inputs may or may not increase with ability. If the government gives a sufficiently high weight to equality, then the optimal policy involves giving more education to those of lower ability than those of higher ability.

Since consumption rises with ability, the marginal utility will eventually fall to $\lambda$ and we enter phase 2 of the solution.

Second interval: $g(n)>0, e(n)>0$ (both public and private education). Here $\phi_{E}=1$ and $u^{\prime}(c)=\lambda$. It follows from the latter condition that consumption is constant on this regime. Since the marginal re- 
turn is constant, it follows that those of higher ability are obtaining more education in total than those of lower ability. But this implies that their gross earnings are higher than those of lower ability. Since their consumption is constant, this implies that private educational outlays are rising just as fast as earnings to leave consumption outlays constant. It follows, further, that public provision of education is being steadily reduced with ability so that this withdrawal of provision is essentially acting as a tax on the individual.

Eventually government provision of education is cut to zero and we enter phase 3 of the solution, corresponding to the highest abilities.

Third interval: $g(n)=0, e(n)>0$ (private education only). Here $\phi_{E}$ $=1$ and $u^{\prime}(c) \phi_{E}<\lambda$, that is, $u^{\prime}(c)<\lambda$. The constant marginal return again implies that those of greater ability spend more on education than those of lesser ability, which implies that they have higher earnings. It is straightforward to show that these rise faster than the expenditure on education, leaving individuals with higher ability a greater amount of consumption.

We see, then, that allowing for the existence of a private market alters Arrow's rules quite considerably. Public education is now to be concentrated at the lower end of the ability range with individuals of very low ability being given more education than they would choose to buy voluntarily on the private market. Individuals of higher ability consider it worthwhile to enter the private market to supplement what is being provided by the state. At this point the systematic withdrawal of public education acts like a tax, and it is now possible to achieve perfect equality. Finally, those with highest ability obtain no public education.

Having studied the effects of the two policies in isolation, let us turn now to the main part of the paper and consider their interaction.

\section{The Two Policies Combined}

In this section we investigate the nature of the optimum policies when the government can choose both the level of education it gives to individuals of different abilities and the income-tax schedule.

Now if the choice of instruments is unrestricted, the government can easily achieve the first-best optimum. For it can set the marginal rate of income tax to 100 percent, thus driving everyone out of the private market, and then allocate government expenditure so as to maximize aggregate consumption which is distributed equally through a uniform lump-sum tax.

This strong and not very interesting conclusion reflects the very simple structure of the model we have been analyzing and would not apply in a more general model which incorporated, for example, the 
possibility that individuals could choose how long they wanted to work at any given wage, and in which 100 percent marginal tax rates would be inappropriate. Consequently, in order to obtain some understanding of how the two policies would interact in a more realistic model, we have chosen to proceed by simply ruling out 100 percent marginal tax rates rather than model directly the factors which would make such tax rates inappropriate. In the light of recent work we have undertaken with more general models, it turns out that the general conclusions we obtain by this ad hoc device are correct.

To understand how we model this constraint on the marginal tax rate, we begin by examining the choice that confronts the individual. An individual of ability $n$ has, once again, to choose the amount of private education he wants to purchase given that he has already received $g(n) \geqq 0$ units of education from the government and confronts an income-tax schedule described by the function $z(w)$. If he seeks to maximize consumption he will

$$
\max _{w \geqq 0}[z(w)+g(n)-\psi(n, w)] \text { such that } \psi(n, w) \geqq g(n) .
$$

The constraint reflects our assumption that the individual may not sell government education.

The solution to this problem is characterized by the first-order conditions

$$
\begin{aligned}
z^{\prime}(w) \leqq \nu(n) \psi_{w}(n, w), & w \geqq 0, \\
\psi(n, w) \leqq g(n), & \nu(n) \leqq 1,
\end{aligned}
$$

$[1-\nu(n)]$ being the Lagrange multiplier on the education constraint, and where both sets of inequalities hold with complementary slackness. Given our assumptions on $\psi$ and the fact that we have ruled out 100 percent marginal tax rates, we can take it that $w>0$, and consequently

$$
z^{\prime}(w)=\nu(n) \psi_{w} .
$$

Once again it turns out to be helpful to characterize the individual's solution in a way that does not contain $z(\cdot)$ explicitly. Accordingly, we differentiate the individual's budget constraint to obtain

$$
\begin{aligned}
c^{\prime}(n) & =z^{\prime}(w) w^{\prime}+g^{\prime}-\psi_{n}-\psi_{w} w^{\prime} \\
& =\nu \psi_{w} w^{\prime}+g^{\prime}-\psi_{n}-\psi_{w} w^{\prime} \\
& =g^{\prime}-\psi_{n}-(1-\nu) \psi_{w} w^{\prime} .
\end{aligned}
$$

Also, since $[1-\nu(n)](\psi-g) \equiv 0$, we find

$$
(1-\nu)\left(\psi_{n}+\psi_{w} w^{\prime}-g^{\prime}\right)=0 .
$$


Thus (27) becomes

$$
c^{\prime}(n)=\nu(n)\left[g^{\prime}(n)-\psi_{n}\right]
$$

Equation (29) is just an extension of (10) and says that along the optimal consumption trajectory, unconstrained individuals of higher ability obtain increases in consumption equal to the increment in government education they receive (which could be negative if $g^{\prime}<0$ ) plus the saving in educational outlay they obtain just by virtue of being more able, while constrained individuals only make a proportion of this gain because they are unable to reduce their private outlays below zero.

Notice also that given (26), we can express the requirement that the marginal tax rate be less than 100 percent by imposing the restriction $\nu(n) \psi_{w}[n, w(n)] \geqq \epsilon>0$. The complete characterization of the government's problem, therefore, is to choose $c(n), g(n), w(n), \nu(n)$ so as to

$$
\begin{gathered}
\max \int_{n}^{\bar{n}} u[c(n)] f(n) d n \\
\text { such that } \int_{n}^{\bar{n}}\{c(n)+\psi[n, w(n)]-w(n)\} f(n) d n \leqq 0, \\
c^{\prime}(n)=\nu(n)\left[g^{\prime}(n)-\psi_{n}\right], \\
\nu(n) \leqq 1, \\
\psi \geqq g, \\
(1-\nu)(\psi-g)=0, \\
\nu \psi_{w} \geqq \epsilon .
\end{gathered}
$$

Here (30) is the overall resource constraint, (31)-(34) give the complete characterization of the individual's optimal choice, and (35) is the restriction on the choice of tax schedule. Introducing Lagrange multipliers $\lambda, \alpha(n), \beta(n), \gamma(n), \pi(n), \sigma(n)$, respectively, we can derive the following first-order conditions for the optimum:

$$
\begin{gathered}
{\left[u^{\prime}(c)-\lambda\right] f+\alpha^{\prime}=0 ;} \\
\lambda\left(1-\psi_{w}\right) f-\alpha \nu \psi_{w w}+[\alpha+\pi(1-\nu)] \psi_{w}+\alpha \nu \psi_{w w}=0 ; \\
\alpha\left(g^{\prime}-\psi_{n}\right)-[\beta+\pi(\psi-g)]+\sigma \psi_{w}=0 ; \\
-\frac{d}{d n}(\alpha \nu)-[\gamma+\pi(1-\nu)] \leqq 0, \quad g(n) \geqq 0 ; \\
\nu \leqq 1, \quad \beta \geqq 0 ; \\
\psi \geqq g, \quad \gamma \geqq 0 ; \\
\nu \psi_{w} \geqq \epsilon, \quad \sigma \geqq 0 .
\end{gathered}
$$

With transversality conditions, $\alpha(\boldsymbol{n})=\alpha(\bar{n})=0$. 
We shall show that once again there are three distinct types of solutions to these conditions. In the first the individual purchases no private education but is allocated some government education. In the second the individual consumes both public and private education, while in the third he consumes only private education.

Given the number of equations and variables involved, it is not possible to determine completely all the characteristics of the optimum solution without direct computation. In particular, we have not been able to determine the precise conditions under which the solution will pass through these various phases. What we shall explore, however, is the nature of a solution which is assumed to pass continuously through all three phases. It will be shown that once again such a solution will divide the ability range into three intervals, people with ability in the lowest interval being in the first phase, and those in the middle interval occupying the second phase. It will turn out to be useful to investigate the intervals in reverse order.

Third interval: Here individuals have only private education. That is, $\psi>g=0$. This implies $\nu \equiv 1, \gamma \equiv 0$. From (39) this implies $\alpha^{\prime} \geqq 0$, and hence $u^{\prime}(c) \leqq \lambda$. On the other hand, (31) now implies $c^{\prime}>0$, so we must have $u^{\prime}(c)<\lambda, \alpha^{\prime}>0$, on the interior of the interval. Moreover, since $\alpha(\bar{n})=0$, it follows from this, and the nature of solutions on the other intervals, that we must have $\alpha<0$ on this interval, and that this interval must cover those in the highest ability range.

If we ignored the tax constraint (35), the solution on this interval would be formally identical to that of a problem of the type considered in Section III. We saw there that marginal tax rates would not reach 100 percent and would certainly be falling at the top of the ability range. Since we can choose how small to set $\epsilon$, it seems reasonable to conclude that the constraint (35) can be chosen so as not to be binding on this interval. All the properties of this interval therefore follow from our discussion in Section III. Consequently, we can turn to an examination of the second interval.

Second interval: Here individuals receive both public and private education. That is, $\psi>g>0$. This implies once again that $\nu \equiv 1, \gamma \equiv$ 0 , but now (39) is an equality and implies $\alpha^{\prime} \equiv 0$. But then, from (36) $u^{\prime}(c)=\lambda$, so consumption, and hence utility, are constant on this interval. It is clear why this is so, for the government withdraws public education from individuals at just the rate at which their requirements for education are falling (assuming they keep their gross income constant). Of course, individuals of higher ability have higher marginal returns from education and so choose to earn more than those of lower ability, but since the net return they obtain on this higher wage is just equal to the additional outlay on education required to increase the wage, there is no gain to consumption. In other 
words, while the direct rate of income tax is less than 100 percent, the combination of direct tax and withdrawal of benefit gives an effective tax rate of 100 percent.

From (37) it follows that the nature of the marginal tax schedule is determined solely by the behavior of $f(\cdot)$ and the cost function $\psi()$. In particular, if the constraint (35) does not hold, it is easy to show that $\operatorname{sign}\left(w z^{\prime \prime} / z\right)=\operatorname{sign}\left[1+\left(n f^{\prime} / f\right)\right]$, so that the qualitative behavior of the marginal tax rate is determined solely by the distribution of abilities.

First interval: Here there is no private education, only public provision. That is, $\psi=g>0$. The first point to establish is that the constraint (35) must bind on this interval. Suppose this were not so, and indeed that $\sigma \equiv 0$ on this interval. From (38) it would follow that

$$
\alpha\left(g^{\prime}-\psi_{n}\right)=\beta \geqq 0 .
$$

But then, from (36) and (31) it is easily shown by drawing a phase diagram that there is no solution satisfying (44) that would start with $\alpha(n)=0$ and end with $\alpha$ negative and $u^{\prime}(c)=\lambda$, which is what would have to be the case if the solution is to be continuous with the second interval. Hence there must be some open subinterval of interval 1 on which $\sigma>0$ and $\nu \psi_{w}=\epsilon$.

We can learn a bit more about this interval by noticing that (37), (38), and (39) can be combined with the expression $g^{\prime}-\psi_{n}=\psi_{w} w^{\prime}$ (which follows from $\psi=g$ ) to give

$$
\lambda\left(1-\psi_{w}\right) f(n)=\frac{d}{d n}\left(\alpha \nu \psi_{w}\right)-\frac{\nu \psi_{w w} \beta}{\psi_{w}} .
$$

In particular, in the subinterval where $\nu \psi_{w}=\epsilon$, this becomes

$$
\lambda\left(1-\psi_{w}\right) f(n)=-\epsilon\left[u^{\prime}(c)-\lambda\right] f(n)-\frac{\beta \mu \psi_{w w}}{\psi_{w}} .
$$

A number of implications now follow:

1. At the junction of intervals 1 and 2 , we must have $g^{\prime}-\psi_{n}=0, \gamma$ $=0, \nu=1$, and hence, from (38), $\beta=\sigma \psi_{w}$. Consequently, either $\beta>0$ and $\sigma>0$ at the end of the interval, or else $\beta=\sigma=0$.

2. It cannot be the case that at the end of the first interval $\nu \psi_{w}>\epsilon$ and $\nu<1$. For suppose these inequalities do both hold; then from (38) it follows, with $\beta=\sigma=0$, that $\alpha\left(g^{\prime}-\psi_{n}\right)=0$. But then we must have $g^{\prime}-\psi_{n} \equiv 0$ at the end of the interval, and so $w^{\prime} \equiv 0$. But then the marginal tax rate must be independent of $n$. That is, $\nu \psi_{w}=k>\epsilon$, where $k$ is a constant; however, from (46), $\left(1-\psi_{w}\right) f(n)=-k\left[u^{\prime}(c)-\right.$ $\lambda$ ]. By continuity with interval 2 , we must have $u^{\prime}(c)=\lambda$, and so $1-$ $\psi_{w}=0$. But then $1-\psi_{w}=1-\nu=\sigma=0$ at the beginning of interval 
2 , and so, from (37), $\alpha=0$ throughout interval 2, which is a contradiction.

3. Nor is it possible that at the end of interval $1, \nu \equiv 1, \nu \psi_{w}>\epsilon$. Suppose this is not so. From (39) it follows that $-\alpha^{\prime}=\gamma \geqq 0$, so $\alpha$ is nonincreasing on this phase; $\alpha^{\prime} \leqq 0$ implies $u^{\prime}(c) \geqq \lambda$. If $u^{\prime}(c)=\lambda$, or $\alpha$ is constant, we obtain the contradiction mentioned above, so suppose $u^{\prime}(c)>\lambda, \alpha^{\prime}<0$. Since $u^{\prime}(c)=\lambda$ on the second interval, it follows that $g^{\prime}-\psi_{n}>0$ at the end of the interval, while since $\alpha$ is negative on interval 2, it must be the case that $\alpha\left(g^{\prime}-\psi_{n}\right)<0$. But (39) yields $\alpha\left(g^{\prime}\right.$ $\left.-\psi_{n}\right)=\beta \geqq 0$ - a contradiction. It follows then that at the end of the first interval we must have $\alpha>0, \beta>0, \nu \equiv 1$.

Notice also that at the beginning of the interval $\alpha(n)=0$, so if $\sigma>0$ at the beginning, $\beta>0$ also. While we have not been able to show that the solution with $\sigma>0, \beta>0$ is the only possibility for the entire interval, it is a possible solution and will certainly characterize the optimal solution toward the end of the interval. Accordingly, we now investigate it in more detail.

From $\psi_{w} \equiv \epsilon$, it follows that

$$
w^{\prime}=-\frac{\psi_{w n}}{\psi_{w w}}>0
$$

while

$$
c^{\prime}=g^{\prime}-\psi_{n}=\psi_{w} w^{\prime}>0 .
$$

So the optimal policy is output and welfare regressive. To see the implications for inputs, we have

$$
g^{\prime}=\psi_{n}-\frac{\psi_{w} \cdot \psi_{w n}}{\psi_{w w}},
$$

which, in general, can take either sign.

For the particular case in which $\psi=n^{-1 / \beta} w^{1 / \beta}$, it is easy to show that

$$
g=(\beta \epsilon)^{1 / 1-\beta} n^{1 / 1-\beta},
$$

so that $g^{\prime}>0$, that is, the education policy is input regressive.

Notice finally that since $\nu \equiv 1$, the individual is receiving from the government just as much education as he would choose to buy for himself.

Having developed this solution in some detail, it is worth summarizing our main findings. As we have just shown, the solution passes through the following stages: Interval $1: g>0, e=0$, lowest range of abilities; Interval 2: $g>0, e>0$, intermediate range of abilities; Interval 3: $g=0, e>0$, highest abilities.

On the first interval, it turns out that the constraint imposed to 
prevent the occurrence of 100 percent marginal rates of income tax must be binding on some open interval of ability levels. When it is binding, the optimal policy is output and welfare regressive. Moreover, at least for the specific functional form used for illustrative purposes, education policy is input regressive.

Uniform utility and consumption are maintained on the second interval. Although the marginal income-tax rate is below 100 percent, the combination of this tax with the gradual withdrawal over the interval of public educational provision produces an effective tax rate of 100 percent. Of course, more able people do earn more, but they generate more tax revenue and pay for more of their own education, the net effect being a constant level of consumption.

Finally, the optimal policy on the third interval is always output regressive, and will normally be input regressive. Thus over the higher ability range, both consumption and private expenditure on education will typically increase with ability.

Overall, therefore, it is clear that the egalitarian nature of the solution will depend critically on the relative size of interval 2 in relation to the other intervals. The combination of tax and public education provision does produce more egalitarian outcomes-and generates higher welfare-than either policy considered alone. Unfortunately, we cannot be more specific about the likely magnitude of the gains involved here without carrying out detailed numerical calculations. Considerations of time and space have prevented us from doing this in the present paper.

\section{Conclusions}

There are, understandably, some major limitations to the model we have been considering in this paper, so that it should be regarded as a very preliminary investigation into the issues raised at the outset. Some of the difficulties, such as fixed labor supply, can be removed at the expense of some additional technical difficulties but without substantially altering the structure of the model or its conclusions (Hare and Ulph 1979).

The most difficult feature of the model to justify, however, is the asymmetrical treatment of the information available to the government. As we indicated in Section I, we can certainly formulate a model in which it is desirable for the government to impose a required minimum purchase of education and to have this minimum level (as well as the tax system) related to observable characteristics, while other characteristics remain unobservable. Suppose that some of the unobservable characteristics affected educational costs. Then for any given observations we would have to solve a problem similar to that in 
Section $\mathrm{V}$ of the paper, except that $g$ could not vary with $n$ (the unobservable element in costs). But as we saw in Section III, it is not at all clear what the structure of private education purchase is like at the bottom end of the ability range. Hence it is not at all clear for what subgroup of the population a given constraint would bind. This makes it quite difficult to say how the minimum level of $g$ should vary with the observable characteristics.

Nevertheless, the analysis of the much simpler model of this paper should prove helpful in understanding and resolving the difficulties of the more general model.

\section{Appendix}

In this Appendix we will show that if the tax schedule is continuous, then it is impossible to have 100 percent marginal tax rates at the bottom end of the tax schedule. Suppose, to the contrary, that we did have 100 percent marginal tax rates on income up to $W^{*}$ and that thereafter the marginal tax rate was less than 100 percent. Such a schedule is shown as $A B Q$ in figure 1. Consider the consumption of an individual of low ability as a function of $W$. This is shown as the curve $A D E$, reflecting the fact that by the time this individual has raised his wage to $W^{*}$, diminishing returns are setting in so fast that his consumption keeps on falling, even though the marginal tax rate is falling. This individual's optimal $W$ is zero. A higher-ability individual may have a consumption curve such as $A F G H$, which again would lead to a choice of zero $W$ even though his consumption rises after $W^{*}$. Clearly as $n$ rises there will be an $n^{*}$ whose consumption curve is just tangent to the extension of the line $A B$. This $n^{*}$ will have the property that all individuals with $n>n^{*}$ will choose to have positive $W$, while those with $n<n^{*}$ will have $W=0$, and utility given by the utility of consumption level $O A$. Thus by choosing the height $O A$ and the distance $O W^{*}$, the government can control the level of ability at which people choose to have a positive wage and the level of utility for those who choose a zero wage.

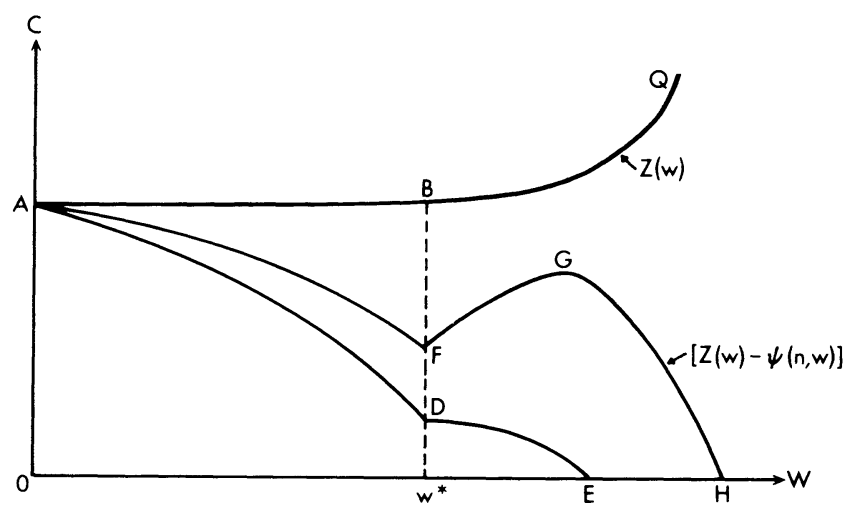

FIG. 1 
The optimal tax problem can now be formulated as the following two-stage problem: Let

$$
\begin{gathered}
V\left(n_{0}, \eta_{0}, R\right) \equiv \max _{\eta(n), w(n)} \int_{n_{0}}^{\bar{n}} \eta(n) f(n) d n \\
\text { such that } \int_{n_{0}}^{\bar{n}}[X(\eta)+\psi(n, w)-w] f(n) d n \leqq R, \\
\eta^{\prime}=-\frac{\psi_{n}}{X_{\eta}}, \\
\eta\left(n_{0}\right)=\eta_{0} .
\end{gathered}
$$

The first-order conditions for this are (12) and (13) in the text, but because of (A3) the transversality conditions are now just $\alpha(\bar{n})=0$.

It is straightforward to show that

$$
\begin{aligned}
& \left.\left.\frac{\partial V}{\partial R}=\lambda \quad \text { (the Lagrange multiplier on [A } 1\right]\right), \\
& \frac{\partial V}{\partial \eta_{0}}=\alpha\left(n_{0}\right), \\
& \frac{\partial V}{\partial n_{0}}=-\eta_{0} f\left(n_{0}\right)+\lambda\left\{X\left(\eta_{0}\right)+\psi\left[n_{0}, w\left(n_{0}\right)\right]-w\left(n_{0}\right)\right\} .
\end{aligned}
$$

The overall problem is to choose $\eta_{0}, n_{0}$ so as to

$$
\max _{\eta_{0}, n_{0}} W\left(\eta_{0}, n_{0}\right) \equiv \eta_{0} F\left(n_{0}\right)+V\left[n_{0}, \eta_{0},-X\left(\eta_{0}\right) F\left(n_{0}\right)\right] .
$$

Clearly

$$
\begin{aligned}
W_{\eta 0} & =\left(1-\lambda X_{\eta}\right) F\left(n_{0}\right)+\alpha\left(n_{0}\right), \\
W_{n 0} & =\eta_{0} f\left(n_{0}\right)-\eta_{0} f\left(n_{0}\right)+\lambda\left\{X\left(\eta_{0}\right)+\psi\left[n_{0}, w\left(n_{0}\right)\right]-w\left(n_{0}\right)\right\}-\lambda X\left(\eta_{0}\right) f\left(n_{0}\right) \\
& =\lambda f\left(n_{0}\right)\left\{\psi\left[n_{0}, w\left(n_{0}\right)\right]-w\left(n_{0}\right)\right\} .
\end{aligned}
$$

On the other hand, we know that the marginal tax rate is positive and less than 100 percent at $w_{0}$, that is,

$$
0<\psi_{w}\left[n_{0}, w\left(n_{0}\right)\right]<1
$$

But given our assumptions on $\psi$, marginal cost must always exceed average cost; hence (A8) implies $\psi\left[n_{0}, w\left(n_{0}\right)\right]<w_{0}$, and so $w_{n 0}<0$ (as long as $n_{0}>\boldsymbol{n}$ ). Hence it can never be optimal to have $n_{0}>\boldsymbol{n}$, and so there is no region of 100 percent marginal tax rates.

\section{References}

$\rightarrow$ Arrow, K. J. "The Utilitarian Approach to the Concept of Equality in Public Expenditure." Q.J.E. 85 (1971): 409-15.

Bruno, M. "Equality, Complementarity and the Incidence of Public Expenditures." J. Public Econ. 6 (1976): 395-407.

Diamond, P. A., and Mirrlees, J. A. "Optimal Taxation and Public Production." A.E.R. 61 (1971): 8-27, 261-78.

$\rightarrow$ Feldstein, N. "On the Optimal Progressivity of the Income Tax." J. Public Econ. 2 (1973): 357-76. 
Hare, P. G., and Ulph, D. T. "The Welfare Economics of Private and Public Education." Paper presented at the conference of the Association of University Teachers of Economics, University of York, March 20-23, 1978.

- "Optimal Taxation and the Allocation of Education." Stirling Univ. Discussion Paper, 1979, in press.

Mirrlees, J. A. "An Exploration in the Theory of Optimum Income Taxation." Rev. Econ. Studies 38 (1971): 175-208.

Roberts, K. "The Treatment of the Poor under Tax/Transfer Schemes." Mimeographed. M.I.T., 1978.

$\rightarrow$ Stern, N. H. "On the Specification of Models of Optimum Income Taxation." J. Public Econ. 6 (1976): 123-62.

Tawney, R. H. Equality. 3d ed. London: Allen \& Unwin, 1938.

Ulph, D. "On the Optimal Distribution of Income and Educational Expenditure." J. Public. Econ. 8 (1977): 341-56. 